\title{
FUZZY TRANSLATIONS AND FUZZY MULTIPLICATIONS OF BCK/BCI-ALGEBRAS
}

\author{
Kyoung Ja Lee, Young Bae Jun, And Myung Im Doh
}

\begin{abstract}
Fuzzy translations, (normalized, maximal) fuzzy extensions and fuzzy multiplications of fuzzy subalgebras in BCK/BCI-algebras are discussed. Relations among fuzzy translations, (normalized, maximal) fuzzy extensions and fuzzy multiplications are investigated.
\end{abstract}

\section{Introduction}

The study of BCK-algebras was initiated by K. Iséki in 1966 as a generalization of the concept of set-theoretic difference and propositional calculus. For the general development of BCK/BCI-algebras, the ideal theory and its fuzzification play an important role. Jun (together with Kim, Meng, Song, and Xin) studied fuzzy trends of several notions in BCK/BCI-algebras (see $[2,3,4,6]$ ). In this paper, we discuss fuzzy translations, (normalized, maximal) fuzzy extensions and fuzzy multiplications of fuzzy subalgebras in BCK/BCI-algebras. We investigate relations among fuzzy translations, (normalized, maximal) fuzzy extensions and fuzzy multiplications.

\section{Preliminaries}

A BCK-algebra is an important class of logical algebras introduced by K. Iséki and was extensively investigated by several researchers.

An algebra $(X ; *, \theta)$ of type $(2,0)$ is called a $B C I$-algebra if it satisfies the following conditions:

(I) $(\forall x, y, z \in X)(((x * y) *(x * z)) *(z * y)=\theta)$,

(II) $(\forall x, y \in X)((x *(x * y)) * y=\theta)$,

(III) $(\forall x \in X)(x * x=\theta)$,

(IV) $(\forall x, y \in X)(x * y=\theta, y * x=\theta \Rightarrow x=y)$.

If a BCI-algebra $X$ satisfies the following identity:

Received November 21, 2008.

2000 Mathematics Subject Classification. 03G25, 06F35, 08A72.

Key words and phrases. fuzzy translation, (normalized, maximal) fuzzy extension, fuzzy multiplication.

This paper has been supported by the 2009 Hannam University Fund. 
(V) $(\forall x \in X)(\theta * x=\theta)$,

then $X$ is called a $B C K$-algebra. Any BCK-algebra $X$ satisfies the following axioms:

(a1) $(\forall x \in X)(x * \theta=x)$

(a2) $(\forall x, y, z \in X)(x * y=\theta \Rightarrow(x * z) *(y * z)=\theta,(z * y) *(z * x)=\theta)$,

(a3) $(\forall x, y, z \in X)((x * y) * z=(x * z) * y)$,

(a4) $(\forall x, y, z \in X)(((x * z) *(y * z)) *(x * y)=\theta)$.

A subset $S$ of a BCK/BCI-algebra $X$ is called a subalgebra of $X$ if $x * y \in S$ for all $x, y \in S$.

We refer the reader to the books [1] and [5] for further information regarding BCK/BCI-algebras.

A fuzzy subset $\mu$ of a BCK/BCI-algebra $X$ is called a fuzzy subalgebra of $X$ if it satisfies:

$$
(\forall x, y \in X)(\mu(x * y) \geq \min \{\mu(x), \mu(y)\}) .
$$

\section{Fuzzy translations and fuzzy multiplications of fuzzy subalgebras}

In what follows let $X=(X, *, \theta)$ denote a BCK/BCI-algebra, and for any fuzzy set $\mu$ of $X$, we denote $\top:=1-\sup \{\mu(x) \mid x \in X\}$ unless otherwise specified.

Definition 3.1. Let $\mu$ be a fuzzy subset of $X$ and let $\alpha \in[0, \top]$. A mapping $\mu_{\alpha}^{T}: X \rightarrow[0,1]$ is called a fuzzy $\alpha$-translation of $\mu$ if it satisfies:

$$
(\forall x \in X)\left(\mu_{\alpha}^{T}(x)=\mu(x)+\alpha\right) .
$$

Theorem 3.2. Let $\mu$ be a fuzzy subalgebra of $X$ and $\alpha \in[0, \top]$. Then the fuzzy $\alpha$-translation $\mu_{\alpha}^{T}$ of $\mu$ is a fuzzy subalgebra of $X$.

Proof. Let $x, y \in X$. Then

$$
\begin{aligned}
\mu_{\alpha}^{T}(x * y) & =\mu(x * y)+\alpha \geq \min \{\mu(x), \mu(y)\}+\alpha \\
& =\min \{\mu(x)+\alpha, \mu(y)+\alpha\}=\min \left\{\mu_{\alpha}^{T}(x), \mu_{\alpha}^{T}(y)\right\} .
\end{aligned}
$$

Hence $\mu_{\alpha}^{T}$ is a fuzzy subalgebra of $X$.

Theorem 3.3. Let $\mu$ be a fuzzy subset of $X$ such that the fuzzy $\alpha$-translation $\mu_{\alpha}^{T}$ of $\mu$ is a fuzzy subalgebra of $X$ for some $\alpha \in[0, \top]$. Then $\mu$ is a fuzzy subalgebra of $X$.

Proof. Assume that $\mu_{\alpha}^{T}$ is a fuzzy subalgebra of $X$ for some $\alpha \in[0, \top]$. Let $x, y \in X$, we have

$$
\begin{aligned}
\mu(x * y)+\alpha & =\mu_{\alpha}^{T}(x * y) \geq \min \left\{\mu_{\alpha}^{T}(x), \mu_{\alpha}^{T}(y)\right\} \\
& =\min \{\mu(x)+\alpha, \mu(y)+\alpha\}=\min \{\mu(x), \mu(y)\}+\alpha
\end{aligned}
$$

which implies that $\mu(x * y) \geq \min \{\mu(x), \mu(y)\}$ for all $x, y \in X$. Hence $\mu$ is a fuzzy subalgebra of $X$. 
Definition 3.4. Let $\mu_{1}$ and $\mu_{2}$ be fuzzy subsets of $X$. If $\mu_{1}(x) \leq \mu_{2}(x)$ for all $x \in X$, then we say that $\mu_{2}$ is a fuzzy extension of $\mu_{1}$.

Definition 3.5. Let $\mu_{1}$ and $\mu_{2}$ be fuzzy subsets of $X$. Then $\mu_{2}$ is called a fuzzy $S$-extension of $\mu_{1}$ if the following assertions are valid:

(i) $\mu_{2}$ is a fuzzy extension of $\mu_{1}$.

(ii) If $\mu_{1}$ is a fuzzy subalgebra of $X$, then $\mu_{2}$ is a fuzzy subalgebra of $X$.

By means of the definition of fuzzy $\alpha$-translation, we know that $\mu_{\alpha}^{T}(x) \geq \mu(x)$ for all $x \in X$. Hence we have the following theorem.

Theorem 3.6. Let $\mu$ be a fuzzy subalgebra of $X$ and $\alpha \in[0, \top]$. Then the fuzzy $\alpha$-translation $\mu_{\alpha}^{T}$ of $\mu$ is a fuzzy $S$-extension of $\mu$.

The converse of Theorem 3.6 is not true in general as seen in the following example.

Example 3.7. Consider a BCK-algebra $X=\{\theta, a, b, c, d\}$ with the following Cayley table:

\begin{tabular}{|l|lllll|}
\hline$*$ & $\theta$ & $a$ & $b$ & $c$ & $d$ \\
\hline$\theta$ & $\theta$ & $\theta$ & $\theta$ & $\theta$ & $\theta$ \\
$a$ & $a$ & $\theta$ & $a$ & $\theta$ & $\theta$ \\
$b$ & $b$ & $b$ & $\theta$ & $b$ & $\theta$ \\
$c$ & $c$ & $a$ & $c$ & $\theta$ & $a$ \\
$d$ & $d$ & $d$ & $d$ & $d$ & $\theta$ \\
\hline
\end{tabular}

Define a fuzzy subset $\mu$ of $X$ by

\begin{tabular}{|c|ccccc|}
\hline$X$ & $\theta$ & $a$ & $b$ & $c$ & $d$ \\
\hline$\mu$ & 0.8 & 0.5 & 0.3 & 0.6 & 0.2 \\
\hline
\end{tabular}

Then $\mu$ is a fuzzy subalgebra of $X$. Let $\nu$ be a fuzzy subset of $X$ given by

\begin{tabular}{|c|ccccc|}
\hline$X$ & $\theta$ & $a$ & $b$ & $c$ & $d$ \\
\hline$\nu$ & 0.84 & 0.56 & 0.38 & 0.67 & 0.21 \\
\hline
\end{tabular}

Then $\nu$ is a fuzzy $S$-extension of $\mu$. But it is not the fuzzy $\alpha$-translation $\mu_{\alpha}^{T}$ of $\mu$ for all $\alpha \in[0, \top]$.

Clearly, the intersection of fuzzy $S$-extensions of a fuzzy subalgebra $\mu$ is a fuzzy $S$-extension of $\mu$. But the union of fuzzy $S$-extensions of a fuzzy subalgebra $\mu$ is not a fuzzy $S$-extension of $\mu$ as seen in the following example. 
Example 3.8. Consider a BCK-algebra $X=\{\theta, a, b, c, d\}$ with the following Cayley table:

\begin{tabular}{|c|ccccc|}
\hline$*$ & $\theta$ & $a$ & $b$ & $c$ & $d$ \\
\hline$\theta$ & $\theta$ & $\theta$ & $\theta$ & $\theta$ & $\theta$ \\
$a$ & $a$ & $\theta$ & $\theta$ & $\theta$ & $\theta$ \\
$b$ & $b$ & $a$ & $\theta$ & $\theta$ & $\theta$ \\
$c$ & $c$ & $a$ & $a$ & $\theta$ & $\theta$ \\
$d$ & $d$ & $c$ & $c$ & $a$ & $\theta$ \\
\hline
\end{tabular}

Define a fuzzy subset $\mu$ of $X$ by

\begin{tabular}{|c|ccccc|}
\hline$X$ & $\theta$ & $a$ & $b$ & $c$ & $d$ \\
\hline$\mu$ & 0.7 & 0.4 & 0.6 & 0.3 & 0.3 \\
\hline
\end{tabular}

Then $\mu$ is a fuzzy subalgebra of $X$. Let $\nu$ and $\delta$ be fuzzy subsets of $X$ given by

\begin{tabular}{|c|ccccc|}
\hline$X$ & $\theta$ & $a$ & $b$ & $c$ & $d$ \\
\hline$\nu$ & 0.8 & 0.6 & 0.8 & 0.4 & 0.4 \\
\hline$\delta$ & 0.9 & 0.6 & 0.6 & 0.6 & 0.7 \\
\hline
\end{tabular}

Then $\nu$ and $\delta$ are fuzzy $S$-extensions of $\mu$. But the union $\nu \cup \delta$ is not a fuzzy $S$-extension of $\mu$ since $(\nu \cup \delta)(d * b)=0.6 \nsupseteq 0.7=\min \{(\nu \cup \delta)(d),(\nu \cup \delta)(b)\}$.

For a fuzzy subset $\mu$ of $X, \alpha \in[0, \top]$ and $t \in[0,1]$ with $t \geq \alpha$, let

$$
U_{\alpha}(\mu ; t):=\{x \in X \mid \mu(x) \geq t-\alpha\} .
$$

If $\mu$ is a fuzzy subalgebra of $X$, then it is clear that $U_{\alpha}(\mu ; t)$ is a subalgebra of $X$ for all $t \in \operatorname{Im}(\mu)$ with $t \geq \alpha$. But, if we do not give a condition that $\mu$ is a fuzzy subalgebra of $X$, then $U_{\alpha}(\mu ; t)$ is not a subalgebra of $X$ as seen in the following example.

Example 3.9. Let $X=\{\theta, a, b, c, d\}$ be a BCK-algebra which is given in Example 3.8. Define a fuzzy subset $\mu$ of $X$ by

\begin{tabular}{|c|ccccc|}
\hline$X$ & $\theta$ & $a$ & $b$ & $c$ & $d$ \\
\hline$\mu$ & 0.7 & 0.4 & 0.6 & 0.3 & 0.5 \\
\hline
\end{tabular}

Then $\mu$ is not a fuzzy subalgebra of $X$ since $\mu(d * b)=0.3 \nsupseteq 0.5=\min \{\mu(d), \mu(b)\}$. For $\alpha=0.1$ and $t=0.5$, we obtain $U_{\alpha}(\mu ; t)=\{\theta, a, b, d\}$ which is not a subalgebra of $X$ since $d * b=c \notin U_{\alpha}(\mu ; t)$.

Theorem 3.10. Let $\mu$ be a fuzzy subset of $X$ and $\alpha \in[0, \top]$. Then the fuzzy $\alpha$-translation $\mu_{\alpha}^{T}$ of $\mu$ is a fuzzy subalgebra of $X$ if and only if $U_{\alpha}(\mu ; t)$ is a subalgebra of $X$ for all $t \in \operatorname{Im}(\mu)$ with $t \geq \alpha$. 
Proof. Necessity is clear. To prove the sufficiency, assume that there exist $a, b \in X$ such that $\mu_{\alpha}^{T}(a * b)<\beta \leq \min \left\{\mu_{\alpha}^{T}(a), \mu_{\alpha}^{T}(b)\right\}$. Then $\mu(a) \geq \beta-\alpha$ and $\mu(b) \geq \beta-\alpha$, but $\mu(a * b)<\beta-\alpha$. This shows that $a, b \in U_{\alpha}(\mu ; \beta)$ and $a * b \notin U_{\alpha}(\mu ; \beta)$. This is a contradiction, and so $\mu_{\alpha}^{T}(x * y) \geq \min \left\{\mu_{\alpha}^{T}(x), \mu_{\alpha}^{T}(y)\right\}$ for all $x, y \in X$. Hence $\mu_{\alpha}^{T}$ is a fuzzy subalgebra of $X$.

Theorem 3.11. Let $\mu$ be a fuzzy subalgebra of $X$ and let $\alpha, \beta \in[0, \top]$. If $\alpha \geq \beta$, then the fuzzy $\alpha$-translation $\mu_{\alpha}^{T}$ of $\mu$ is a fuzzy $S$-extension of the fuzzy $\beta$-translation $\mu_{\beta}^{T}$ of $\mu$.

Proof. Straightforward.

For every fuzzy subalgebra $\mu$ of $X$ and $\beta \in[0, \top]$, the fuzzy $\beta$-translation $\mu_{\beta}^{T}$ of $\mu$ is a fuzzy subalgebra of $X$. If $\nu$ is a fuzzy $S$-extension of $\mu_{\beta}^{T}$, then there exists $\alpha \in[0, \top]$ such that $\alpha \geq \beta$ and $\nu(x) \geq \mu_{\alpha}^{T}(x)$ for all $x \in X$. Thus we have the following theorem.

Theorem 3.12. Let $\mu$ be a fuzzy subalgebra of $X$ and $\beta \in[0, \top]$. For every fuzzy $S$-extension $\nu$ of the fuzzy $\beta$-translation $\mu_{\beta}^{T}$ of $\mu$, there exists $\alpha \in[0, \top]$ such that $\alpha \geq \beta$ and $\nu$ is a fuzzy $S$-extension of the fuzzy $\alpha$-translation $\mu_{\alpha}^{T}$ of $\mu$.

The following example illustrates Theorem 3.12.

Example 3.13. Consider a BCK-algebra $X=\{\theta, a, b, c, d\}$ with the following Cayley table:

$$
\begin{array}{|l|lllll|}
\hline * & \theta & a & b & c & d \\
\hline \theta & \theta & \theta & \theta & \theta & \theta \\
a & a & \theta & \theta & a & a \\
b & b & b & \theta & b & b \\
c & c & c & c & \theta & c \\
d & d & d & d & d & \theta \\
\hline
\end{array}
$$

Define a fuzzy subset $\mu$ of $X$ by

\begin{tabular}{|c|ccccc|}
\hline$X$ & $\theta$ & $a$ & $b$ & $c$ & $d$ \\
\hline$\mu$ & 0.7 & 0.4 & 0.2 & 0.5 & 0.1 \\
\hline
\end{tabular}

Then $\mu$ is a fuzzy subalgebra of $X$, and $T=0.3$. If we take $\beta=0.2$, then the fuzzy $\beta$-translation $\mu_{\beta}^{T}$ of $\mu$ is given by

\begin{tabular}{|c|ccccc|}
\hline$X$ & $\theta$ & $a$ & $b$ & $c$ & $d$ \\
\hline$\mu_{\beta}^{T}$ & 0.9 & 0.6 & 0.4 & 0.7 & 0.3 \\
\hline
\end{tabular}

Let $\nu$ be a fuzzy subset of $X$ defined by

\begin{tabular}{|c|ccccc|}
\hline$X$ & $\theta$ & $a$ & $b$ & $c$ & $d$ \\
\hline$\nu$ & 0.94 & 0.63 & 0.55 & 0.88 & 0.37 \\
\hline
\end{tabular}


Then $\nu$ is clearly a fuzzy subalgebra of $X$ which is fuzzy extension of $\mu_{\beta}^{T}$, and hence $\nu$ is a fuzzy $S$-extension of the fuzzy $\beta$-translation $\mu_{\beta}^{T}$ of $\mu$. But $\nu$ is a not a fuzzy $\alpha$-translation of $\mu$ for all $\alpha \in[0, \top]$. Take $\alpha=0.23$. Then $\alpha=0.23>0.2=\beta$, and the fuzzy $\alpha$-translation $\mu_{\alpha}^{T}$ of $\mu$ is given as follows:

\begin{tabular}{|c|ccccc|}
\hline$X$ & $\theta$ & $a$ & $b$ & $c$ & $d$ \\
\hline$\mu_{\alpha}^{T}$ & 0.93 & 0.63 & 0.43 & 0.73 & 0.33 \\
\hline
\end{tabular}

Note that $\nu(x) \geq \mu_{\alpha}^{T}(x)$ for all $x \in X$, and hence $\nu$ is a fuzzy $S$-extension of the fuzzy $\alpha$-translation $\mu_{\alpha}^{T}$ of $\mu$.

A fuzzy $S$-extension $\nu$ of a fuzzy subalgebra $\mu$ of $X$ is said to be normalized if there exists $x_{0} \in X$ such that $\nu\left(x_{0}\right)=1$. Let $\mu$ be a fuzzy subalgebra of $X$. A fuzzy subset $\nu$ of $X$ is called a maximal fuzzy $S$-extension of $\mu$ if it satisfies:

(i) $\nu$ is a fuzzy $S$-extension of $\mu$,

(ii) there does not exist another fuzzy subalgebra of $X$ which is a fuzzy extension of $\nu$.

Example 3.14. Let $\mathbb{N}$ be the set of all natural numbers and let $*$ be a binary operation on $\mathbb{N}$ defined by

$$
(\forall a, b \in \mathbb{N})\left(a * b=\frac{a}{(a, b)}\right),
$$

where $(a, b)$ is the greatest common divisor of $a$ and $b$. Then $(\mathbb{N} ; *, 1)$ is a BCKalgebra. Let $\mu$ and $\nu$ be fuzzy subsets of $\mathbb{N}$ which are defined by $\mu(x)=\frac{1}{3}$ and $\nu(x)=1$ for all $x \in \mathbb{N}$. Clearly $\mu$ and $\nu$ are fuzzy subalgebras of $\mathbb{N}$. It is easy to verify that $\nu$ is a maximal fuzzy $S$-extension of $\mu$.

Proposition 3.15. If a fuzzy subset $\nu$ of $X$ is a normalized fuzzy $S$-extension of a fuzzy subalgebra $\mu$ of $X$, then $\nu(\theta)=1$.

Proof. It is clear because $\nu(\theta) \geq \nu(x)$ for all $x \in X$.

Theorem 3.16. Let $\mu$ be a fuzzy subalgebra of $X$. Then every maximal fuzzy $S$-extension of $\mu$ is normalized.

Proof. This follows from the definitions of the maximal and normalized fuzzy $S$-extensions.

Definition 3.17. Let $\mu$ be a fuzzy subset of $X$ and $\gamma \in[0,1]$. A fuzzy $\gamma$ multiplication of $\mu$, denoted by $\mu_{\gamma}^{m}$, is defined to be a mapping

$$
\mu_{\gamma}^{m}: X \rightarrow[0,1], x \mapsto \mu(x) \cdot \gamma
$$

For any fuzzy subset $\mu$ of $X$, a fuzzy 0 -multiplication $\mu_{0}^{m}$ of $\mu$ is clearly a fuzzy subalgebra of $X$.

Theorem 3.18. If $\mu$ is a fuzzy subalgebra of $X$, then the fuzzy $\gamma$-multiplication of $\mu$ is a fuzzy subalgebra of $X$ for all $\gamma \in[0,1]$.

Proof. Straightforward. 
Theorem 3.19. For any fuzzy subset $\mu$ of $X$, the following are equivalent:

(i) $\mu$ is a fuzzy subalgebra of $X$.

(ii) $(\forall \gamma \in(0,1])\left(\mu_{\gamma}^{m}\right.$ is a fuzzy subalgebra of $\left.X\right)$.

Proof. Necessity follows from Theorem 3.18. Let $\gamma \in(0,1]$ be such that $\mu_{\gamma}^{m}$ is a fuzzy subalgebra of $X$. Then

$$
\begin{aligned}
\mu(x * y) \cdot \gamma & =\mu_{\gamma}^{m}(x * y) \geq \min \left\{\mu_{\gamma}^{m}(x), \mu_{\gamma}^{m}(y)\right\} \\
& =\min \{\mu(x) \cdot \gamma, \mu(y) \cdot \gamma\}=\min \{\mu(x), \mu(y)\} \cdot \gamma
\end{aligned}
$$

for all $x, y \in X$, and so $\mu(x * y) \geq \min \{\mu(x), \mu(y)\}$ for all $x, y \in X$ since $\gamma \neq 0$. Hence $\mu$ is a fuzzy subalgebra of $X$.

Theorem 3.20. Let $\mu$ be a fuzzy subset of $X, \alpha \in[0, \top]$ and $\gamma \in(0,1]$. Then every fuzzy $\alpha$-translation $\mu_{\alpha}^{T}$ of $\mu$ is a fuzzy $S$-extension of the fuzzy $\gamma$-multiplication $\mu_{\gamma}^{m}$ of $\mu$.

Proof. For every $x \in X$, we have $\mu_{\alpha}^{T}(x)=\mu(x)+\alpha \geq \mu(x) \geq \mu(x) \cdot \gamma=\mu_{\gamma}^{m}(x)$, and so $\mu_{\alpha}^{T}$ is a fuzzy extension of $\mu_{\gamma}^{m}$. Assume that $\mu_{\gamma}^{m}$ is a fuzzy subalgebra of $X$. Then $\mu$ is a fuzzy subalgebra of $X$ by Theorem 3.19. It follows from Theorem 3.2 that $\mu_{\alpha}^{T}$ is a fuzzy subalgebra of $X$ for all $\alpha \in[0, \top]$. Hence every fuzzy $\alpha$-translation $\mu_{\alpha}^{T}$ is a fuzzy $S$-extension of the fuzzy $\gamma$-multiplication $\mu_{\gamma}^{m}$.

The following example shows that Theorem 3.20 is not valid for $\gamma=0$.

Example 3.21. Consider a BCI-algebra $(\mathbb{Z}, *, 0)$ where $\mathbb{Z}$ is the set of all integers and $*$ is the minus operation. Define a fuzzy set $\mu: \mathbb{Z} \rightarrow[0,1]$ by

$$
\mu(x):= \begin{cases}0 & \text { if } x>0 \\ \frac{1}{2} & \text { if } x \leq 0 .\end{cases}
$$

Taking $\gamma=0$, we see that $\mu_{0}^{m}(x * y)=0=\min \left\{\mu_{0}^{m}(x), \mu_{0}^{m}(y)\right\}$ for all $x, y \in \mathbb{Z}$, that is, $\mu_{0}^{m}$ is a fuzzy subalgebra of $\mathbb{Z}$. But if we take $x=-3$ and $y=-5$, then $\mu_{\alpha}^{T}(x * y)=\mu_{\alpha}^{T}(2)=\mu(2)+\alpha=\alpha<\frac{1}{2}+\alpha=\min \left\{\mu_{\alpha}^{T}(x), \mu_{\alpha}^{T}(y)\right\}$ for all $\alpha \in\left[0, \frac{1}{2}\right]$. Hence $\mu_{\alpha}^{T}$ is not a fuzzy $S$-extension of $\mu_{0}^{m}$ for all $\alpha \in\left[0, \frac{1}{2}\right]$.

The following example illustrates Theorem 3.20.

Example 3.22. Let $X=\{\theta, a, b, c, d\}$ be a BCK-algebra which is given in Example 3.13, and consider a fuzzy subalgebra $\mu$ of $X$ that is defined in Example 3.13. If we take $\gamma=0.1$, then the fuzzy $\gamma$-multiplication $\mu_{0.1}^{m}$ of $\mu$ is given by

\begin{tabular}{|c|ccccc|}
\hline$X$ & $\theta$ & $a$ & $b$ & $c$ & $d$ \\
\hline$\mu_{0.1}^{m}$ & 0.07 & 0.04 & 0.02 & 0.05 & 0.01 \\
\hline
\end{tabular}


Clearly $\mu_{0.1}^{m}$ is a fuzzy subalgebra of $X$. Also, for any $\alpha \in[0,0.3]$, the fuzzy $\alpha$-translation $\mu_{\alpha}^{T}$ of $\mu$ is given by

\begin{tabular}{|c|ccccc|}
\hline$X$ & $\theta$ & $a$ & $b$ & $c$ & $d$ \\
\hline$\mu_{\alpha}^{T}$ & $0.7+\alpha$ & $0.4+\alpha$ & $0.2+\alpha$ & $0.5+\alpha$ & $0.1+\alpha$ \\
\hline
\end{tabular}

Then $\mu_{\alpha}^{T}$ is a fuzzy extension of $\mu_{0.1}^{m}$ and $\mu_{\alpha}^{T}$ is always a fuzzy subalgebra of $X$ for all $\alpha \in[0,0.3]$. Hence $\mu_{\alpha}^{T}$ is a fuzzy $S$-extension of $\mu_{0.1}^{m}$ for all $\alpha \in[0,0.3]$.

\section{References}

[1] Y. Huang, BCI-algebra, Science Press, Beijing, 2006.

[2] Y. B. Jun and J. Meng, Fuzzy commutative ideals in BCI-algebras, Commun. Korean Math. Soc. 9 (1994), no. 1, 19-25.

[3] Y. B. Jun and S. Z. Song, Fuzzy set theort applied to implicative ideals in BCK-algebras, Bull. Korean Math. Soc. 43 (2006), no. 3, 461-470.

[4] Y. B. Jun and X. L. Xin, Involutory and invertible fuzzy BCK-algebras, Fuzzy Sets and Systems 117 (2004), 463-469.

[5] J. Meng and Y. B. Jun, BCK-algebras, Kyungmoon Sa Co. Seoul, 1994.

[6] J. Meng, Y. B. Jun, and H. S. Kim, Fuzzy implicative ideals of BCK-algebras, Fuzzy Sets and Systems 89 (1997), 243-248.

Kyoung Ja LeE

Department of Mathematics Education

HANNAM UNIVERSITY

DAEJEON 306-791, KoreA

E-mail address: kjlee@hnu.kr

Young Bae Jun

Department of Mathematics Education (and Rins)

Gyeongsang National University

Chinju 660-701, Korea

E-mail address: skywine@gmail.com, ybjun@gnu.kr

Myung Im DoH

Department of Mathematics

Gyeongsang National University

Chinju 660-701, Korea

E-mail address: sansudo6@hanmail.net 